\title{
Long-Term Survival After Composite Mechanical Aortic Root Replacement
}

\author{
Khaled E. Al-Ebrahim, FRCSC* , Husain H. Jabbad, FRCSC, Ahmad H. Alqari, MBBS \\ Division of Cardiothoracic Surgery, King Abdulaziz University Hospital, Jeddah, Saudi Arabia
}

\begin{abstract}
This report describes the long-term follow-up of the repair of a giant ascending aneurysm using a composite graft with a mechanical valve.

Copyright @ 2016 Science International Corp.
\end{abstract}

\section{Key Words}

Aneurysm • Aortic • Ascending • Composite root

A 55-year-old male presented with severe cardiac and renal failure ten years ago. Electrocardiogram (ECG) showed aortic root dilatation, severe aortic regurgitation, and left ventricular dysfunction. Chest computed tomography revealed an ascending aortic aneurysm larger than the heart, measuring $13.5 \mathrm{~cm}$ in the transverse diameter and $12 \mathrm{~cm}$ anteroposteriorly (Figure 1). Aortic root replacement using a size 33 composite graft and coronary reimplantation were performed. Ejection fraction, renal function, and general health gradually improved. This patient was followed-up for 10 years with no complications and has led a normal life. Recent chest computed tomography showed normal aortic root and coronary arteries (Figure 2). Right coronary implantation is shown in a colored three-dimensional image (Figure 3). This case demonstrates excellent longevity and freedom from reoperation in patients who undergo composite root replacement.

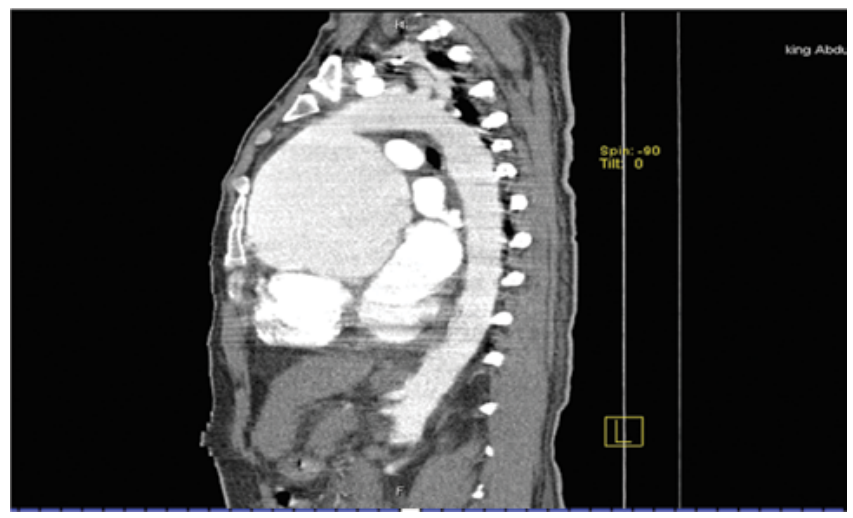

Figure 1. Preoperative computed tomography image showing a $13-\mathrm{cm}$ ascending aortic aneurysm involving the sinuses of Valsalva.

\author{
(c) 2016 AORTA \\ Published by Science International Corp. \\ ISSN 2325-4637 \\ Accessible online at: \\ http://aorta.scienceinternational.org
}

* Corresponding Author:

Khalid E. Al-Ebrahim, FRCSC Division of Cardiothoracic Surgery King Abdulaziz University Hospital PO Box 80215, Jeddah 21589, Saudi Arabia

Tel.: +9662 640 1000; Fax: +9662 640 1238; E-Mail: dr.k.ebrahim@gmail.com 


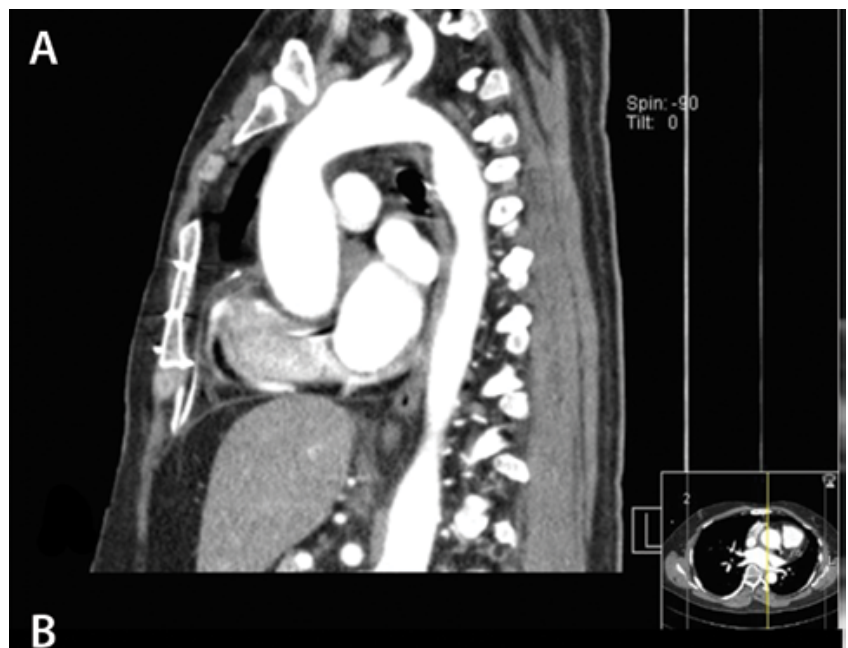

B

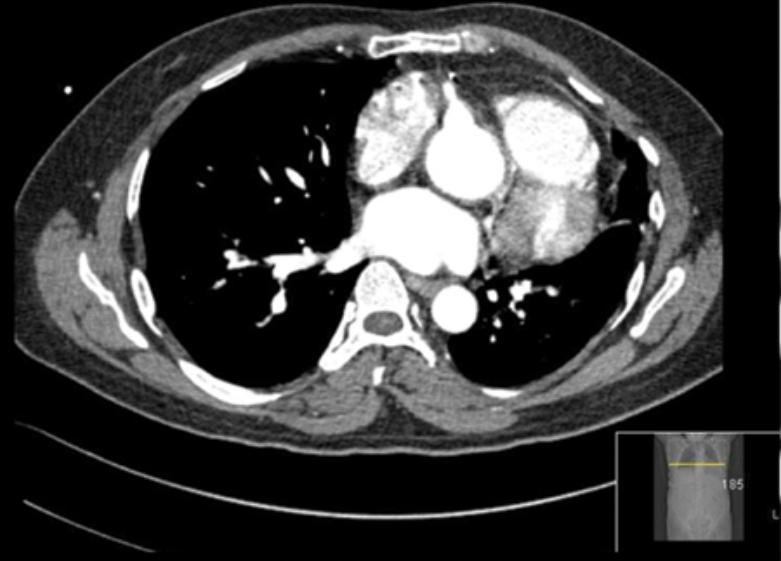

C

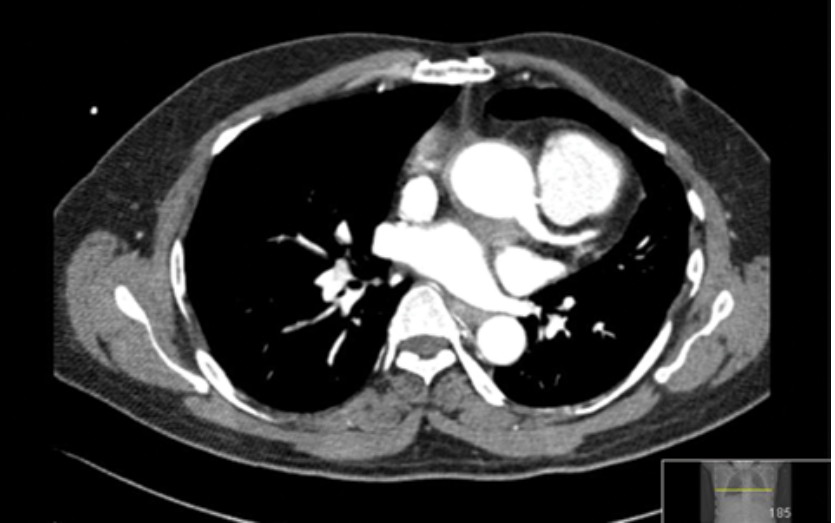

Figure 2. Postoperative computed tomography images obtained 10 years after surgery. Panel $A$. Sagittal section showing the neoascending aorta. Panel $B$. Coronal section showing the neo-right coronary artery. Panel C. Coronal section showing the neo-left coronary artery.

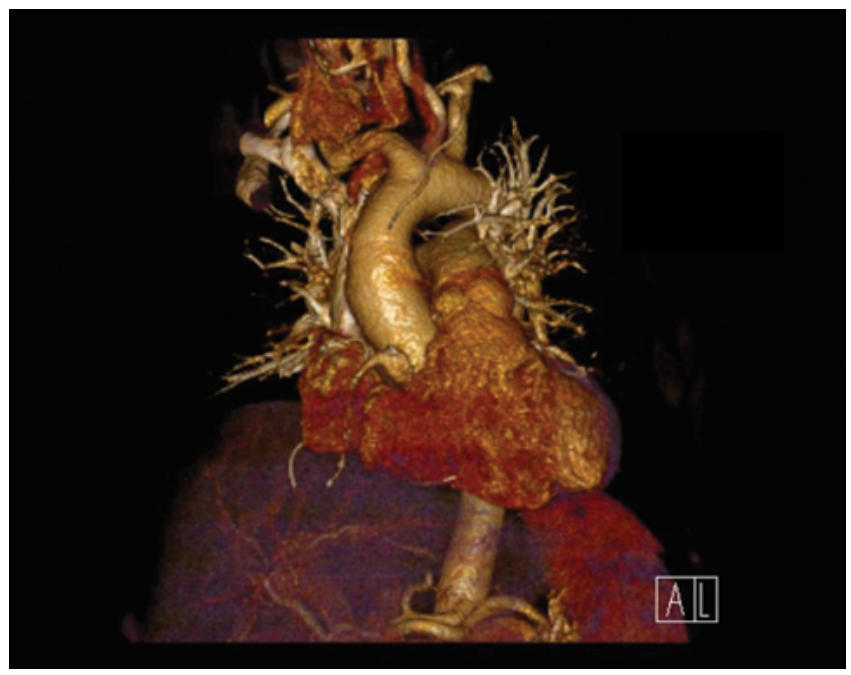

Figure 3. Postoperative computed tomography image obtained 10 years after surgery showing the ascending aorta and right coronary artery.

\section{Conflict of Interest}

The authors have no conflict of interest relevant to this publication.

\section{Comment on this Article or Ask a Question}

Cite this article as: Al-Ebrahim KE, Jabbad HH, Alqari AH. LongTerm Survival After Composite Mechanical Aortic Root Replacement. AORTA (Stamford). 2016;4(4):146-147. DOI: http://dx.doi.org/10.12945/j.aorta.2016.15.039 